\title{
Keck Planet Imager and Characterizer: concept and phased implementation
}

D. Mawet ${ }^{a, b}$, P. Wizinowich ${ }^{c}$, R. Dekany ${ }^{a}$, M. Chun ${ }^{d}$, D. Hall ${ }^{d}$, S. Cetre ${ }^{c}$, O. Guyon ${ }^{e}$, J.K. Wallace $^{b}$, B. Bowler ${ }^{f}$, M. Liu ${ }^{g}$, G. Ruane ${ }^{a}$, E. Serabyn ${ }^{b}$, R. Bartos ${ }^{b}$, J. Wang $^{a}$, G. Vasisht ${ }^{b}$, M. Fitzgerald $^{h}$, A. Skemer ${ }^{i}$, M. Ireland ${ }^{j}$, J. Fucik ${ }^{a}$, J. Fortney ${ }^{i}$, I. Crossfield ${ }^{i, k}$, R. Hu ${ }^{b}$, B. Benneke $^{a}$

${ }^{a}$ Department of Astronomy, California Institute of Technology, Pasadena, CA 91106, USA;

${ }^{b}$ Jet Propulsion Laboratory, California Institute of Technology, Pasadena, CA 91109, USA;

${ }^{c}$ W. M. Keck Observatory, 65-1120 Mamalahoa Hwy., Kamuela, HI, USA 96743;

${ }^{d}$ Institute for Astronomy, University of Hawaii, 640 N. Aóhoku Place, Hilo, HI, USA 96720;

${ }^{e}$ Subaru Telescope, National Astronomical Observatory of Japan,

650 N, Aóhoku Place, Hilo, HI, USA 96720;

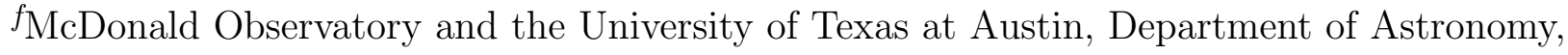
2515 Speedway, Stop C1400, Austin, TX 78712;

${ }^{g}$ Institute for Astronomy, University of Hawaii, 2680 Woodlawn Drive, Honolulu, HI, USA 96822

${ }^{h}$ Department of Physics and Astronomy, University of California-Los Angeles, 430 Portola Plaza, Los Angeles, CA 90095, USA;

${ }^{i}$ Department of Astronomy and Astrophysics, University of California-Santa Cruz, Santa Cruz, CA 95064, USA;

${ }^{j}$ Research School of Astronomy and Astrophysics, Australian National University,

Canberra, ACT 2611, Australia;

${ }^{k}$ Lunar \& Planetary Laboratory, University of Arizona, 1629 E. University Blvd., Tucson, AZ, USA.

\begin{abstract}
The Keck Planet Imager and Characterizer (KPIC) is a cost-effective upgrade path to the W.M. Keck observatory (WMKO) adaptive optics (AO) system, building on the lessons learned from first and second-generation extreme AO (ExAO) coronagraphs. KPIC will explore new scientific niches in exoplanet science, while maturing critical technologies and systems for future ground-based (TMT, EELT, GMT) and space-based planet imagers (HabEx, LUVOIR). The advent of fast low-noise IR cameras (IR-APD, MKIDS, electron injectors), the rapid maturing of efficient wavefront sensing (WFS) techniques (Pyramid, Zernike), small inner working angle (IWA) coronagraphs (e.g., vortex) and associated low-order wavefront sensors (LOWFS), as well as recent breakthroughs in high contrast high resolution spectroscopy, open new direct exoplanet exploration avenues that are complementary to planet imagers such as VLT-SPHERE and the Gemini Planet Imager (GPI). For instance, the search and detailed characterization of planetary systems on solar-system scales around late-type stars, mostly beyond SPHERE and GPI's reaches, can be initiated now at WMKO.
\end{abstract}

Keywords: Exoplanets, high contrast imaging, high contrast high resolution spectroscopy, small inner working angle coronagraphy, vortex coronagraph, on-axis segmented telescopes, apodization, Extremely Large Telescopes

Further author information: send correspondence to dmawet@astro.caltech.edu 

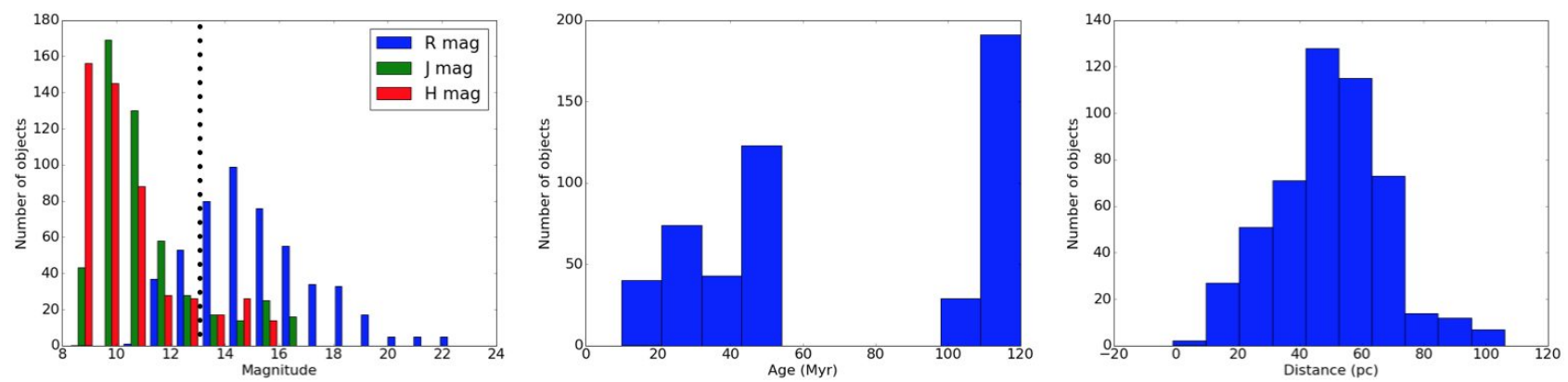

Figure 1. Left: Distribution of R, J, H magnitudes for a notional survey sample of 500 young, close-by late-type stars. The dashed line represents the cutoff natural guide star (NGS) brightness for L-band high contrast imaging. This plots shows the dramatic increase in sky coverage enabled by IR wavefront sensing: many more red targets can be addressed below the NGS magnitude cutoff in the IR. Middle: Age distribution (Myr) of our notional sample. Right: Distance distribution (pc) for the same notional sample.

\section{INTRODUCTION}

In 2014, we submitted the Keck Planet Imager and Characterizer concept to the Keck Science Steering Committee (KSSC) in response to a call for instrument white papers. The proposed KPIC concept consists of an insertable/removable module downstream from the current Keck AO system, equipped with its own efficient IR wavefront sensor (IR WFS) using the latest low-noise detector technologies (IR-APD), a high-order deformable mirror (HODM), a state-of-the-art coronagraphic bench, and a beamsplitter dividing the science beam into an imaging path and a fiber-injection unit (FIU). Both channels are available to feed existing IR science instruments available at Keck: the imager NIRC2, the IFU OSIRIS (or another IFU yet to come) for the (spectro-)imaging channels, and the high-resolution IR spectrograph NIRSPEC for the fiber-fed spectroscopic channel.

An extreme adaptive optics (ExAO) high contrast imaging facility optimized for faint red objects by means of IR wavefront sensing can image and spectroscopically characterize exoplanets both around nearby young $\mathrm{K}$ and $\mathrm{M}$ stars, and infant planetary systems in distant and obscured star-forming regions. KPIC will provide the WMKO community with new capabilities orthogonal to those at Subaru, VLT, Gemini, and LBT.

\section{KPIC SCIENCE CASES}

Exoplanets around late-type stars. M dwarfs constitute a promising reservoir to survey in order to advance our understanding of planetary formation and evolution. Indeed, $\mathrm{M}$ dwarfs outnumber all earlier-type stars together. ${ }^{1}$ Their abundance, low close binary fraction, and the ubiquitous presence of massive protoplanetary disks at young ages imply that they are common sites of planet formation. Close separations $(<1 \mathrm{AU})$ have been extensively probed by Doppler and transit surveys with the following results: the frequency of close-in giant planets $\left(1-10 M_{J u p}\right)$ is only $2.5 \pm 0.9 \%$, consistent with core accretion plus migration models. ${ }^{2}$ On the other hand, the Kepler survey indicates that Earth- to Neptune-sized planets might be as common as one per star. $^{3-5}$ The outskirts of young M-dwarf systems $(10-100 \mathrm{AU})$ have been probed by first-generation direct imaging instruments, and results show that massive planets are rare: fewer than $10.6 \%$ of M-dwarf systems surveyed harbor $1-13 M_{\text {Jup }}$ giant planets in their outer regions. ${ }^{6}$ Disk instability does not seem to be a common mechanism of giant planet formation. The 1-10 AU parameter space is thus believed to be the overwhelmingly favored region for planet formation. Across the entire range of sensitivity $\left(10 M_{\oplus}-10 M_{J u p}\right)$, the occurrence rates measured by microlensing surveys imply an average $1.6_{-0.9}^{+0.7}$ planets per star ${ }^{7}$ ! Microlensing probes the full range of planetary masses in this region, but the masses and metallicities of the host stars are usually poorly constrained and so are of limited value for statistical studies. Moreover, one-shot microlensing-detected objects can not be followed up. High contrast imaging with a good knowledge of the host star distance and age is therefore the perfect complement to indirect techniques, and holds the promise of filling in this untouched parameter space, and provide excellent characterization opportunities. Last but not least, $\mathrm{M}$ dwarfs provide the best star-planet contrast ratios for young giant planets among all stellar masses. 


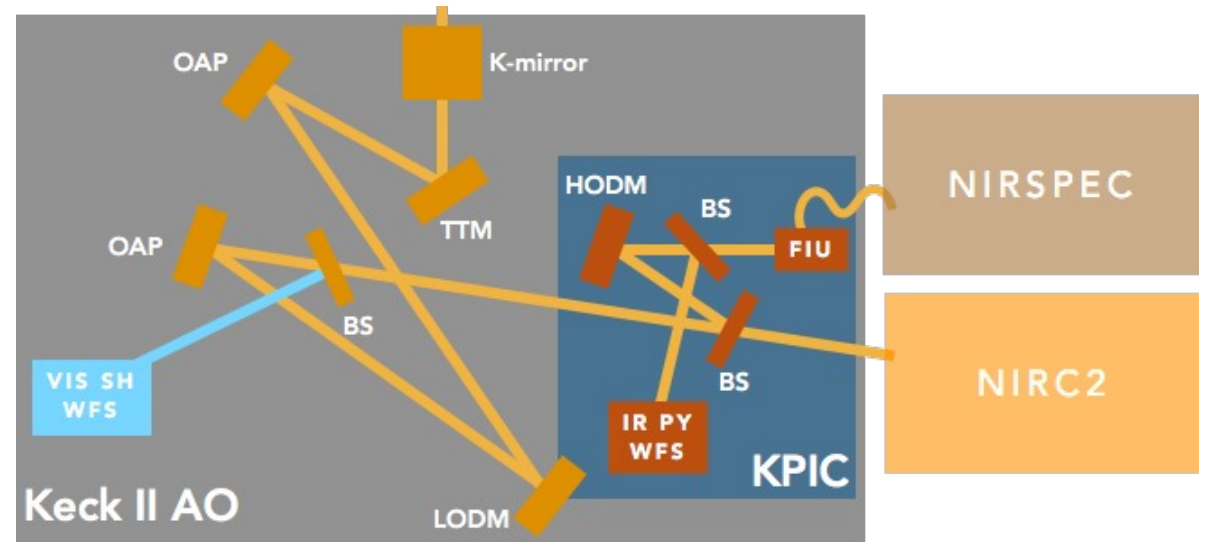

Figure 2. Conceptual layout of KPIC, as integrated on the Keck II AO bench, and feeding IR workhorse camera NIRC2, and high-resolution IR spectrograph NIRSPEC, by means of a fiber injection unit (FIU).

KPIC will target this reservoir (Fig. 1) with the most advanced high contrast imaging technologies, unveiling these planets with unprecedented sensitivity, but also directly analyzing their thermal light with high-resolution spectroscopy (NIRSPEC), enabling the retrieval of the molecular composition of their atmosphere ${ }^{8}$ and in favorable cases the Doppler imaging of cloud, wind, and temperature gradients. ${ }^{9-11}$ See J. Wang et al. 2016 (these proceedings) for more details.

Planetary systems in star forming regions (SFR). Sky coverage with an IR WFS is typically $50 \%$ higher than with a classical visible WFS. ${ }^{12}$ In obscured areas such as SFR, the gain is much more dramatic. The population of young stars in Taurus, $140 \mathrm{pc}$ away, is dominated by $\mathrm{M}$ stars and very late $\mathrm{K}$ stars ${ }^{13}$ making IR WFS essential for these very red stars. Indeed, an R-band WFS sensitivity rolloff at $R \simeq 10$ currently provides access to only a handful of $\mathrm{T}$ Tauri stars, while a rolloff at $J / H \simeq 10$ mags would enable high contrast on a hundred young stars in Taurus alone. Thus, IR wavefront sensing enables high contrast imaging studies of extrasolar planetary systems (both disk + protoplanets) in their infancy.

Galactic center (GC). IR WFS has proven to be very robust to study the GC with VLT-NACO: IRS 7 $(H=9.3, K=6.5)$ is only 6" North of $S g r A *$. An IR WFS ExAO would boost the Strehl ratio (SR) by a factor of a few compared to current LGS assisted observations (10-30\% SR at K), therefore enhancing SNR, enabling somewhat shorter wavelengths (flux quickly drops off as the huge extinction towards the GC kicks in), and thus improving resolution and astrometric precision (reduced confusion).

\section{KPIC CONCEPT AND PHASED IMPLEMENTATION}

The technological landscape has evolved considerably since powerhouse second-generation AO systems such as SPHERE and GPI were designed, in particular in wavefront sensing, coronagraphic, and detector technologies. KPIC takes full advantage of the latest technology developments in these areas. Specifically, we will supplement the current Keck-AO system with a cascaded IR Pyramid WFS (IR PWFS) ExAO system (P. Wizinowich et al. 2016, these proceedings) using a state-of-the-art fast, low read-noise IR APD array from SELEX (SAPHIRA: D. Atkinson et al. 2016, D. Hall et al. 2016, J.L. Gach et al. 2016, these proceedings), and high-order deformable mirror. The new proposed module maximizes the use of current Keck infrastructure and assets (Fig. 2). New developments are kept to a minimum, and focussed on key areas which are highly strategic to future TMT planet imager technologies.

The implementation of KPIC proceeds by incremental, non-invasive phases, minimizing disruption of operations, and guaranteeing rapid science return: 

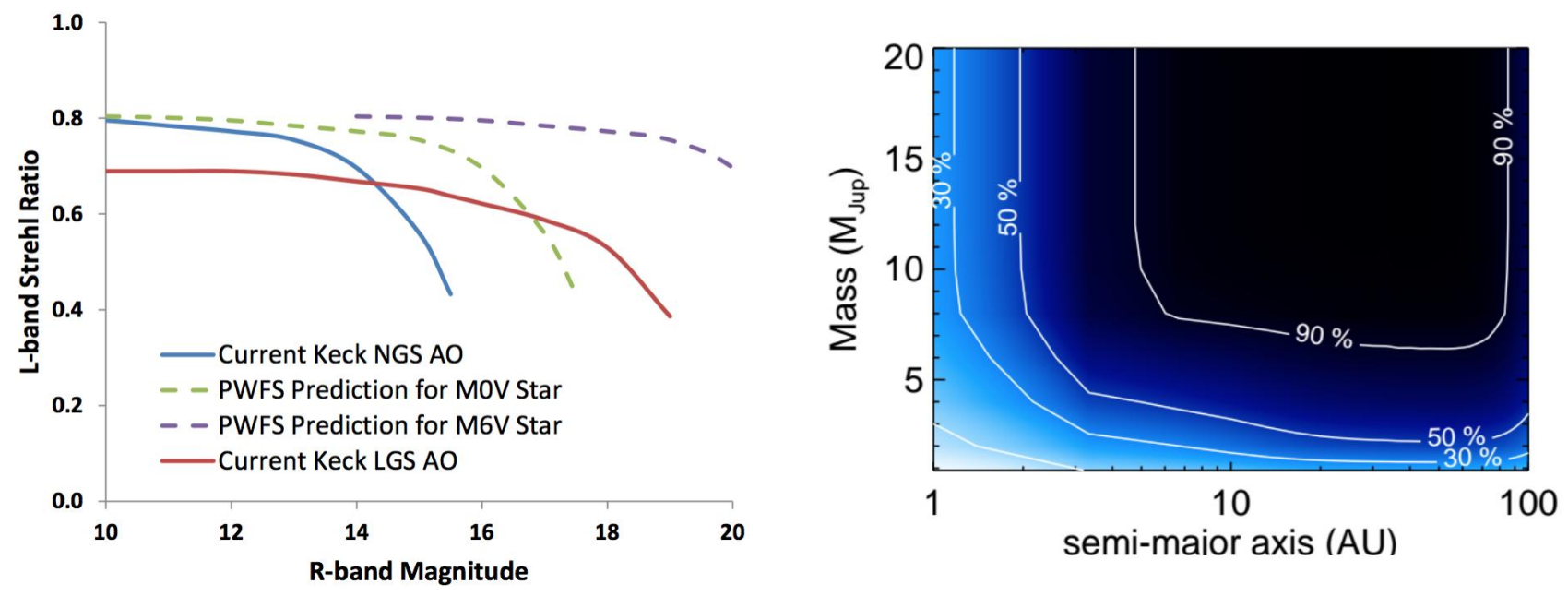

Figure 3. Left: Current and predicted L-band Strehl ratios versus the R-magnitude of an on-axis guide star under median conditions. The current Keck NGS AO system uses a visible Shack-Hartmann WFS. The predicted NGS AO performance is for an efficient low-noise IR PWFS using M0V and M6V spectral types guide stars, respectively. Right: Completeness of the proposed survey, assuming the AO performance shown on the left and our performance model for the recently commissioned L-band vortex coronagraph.

1. Phase I: Installation of a couple high-performance small inner working angle L-band vortex coronagraphs in NIRC2 (B. Femenia et al. 2016 and O. Absil et al. 2016, these proceedings). As part of this coronagraphic upgrade, our team has also developed critically important and game-changing image plane pointing and wavefront control techniques (E. Huby et al. 2016 and M. Bottom et al. 2016, these proceedings). See also Serabyn et al. 2016 (submitted to ApJ).

2. Phase II: Implementation of an IR Pyramid WFS to improve AO correction and sky coverage (P. Wizinowich et al. 2016, these proceedings).

3. Phase III: Fiber link from Keck-AO to IR high-resolution spectrograph NIRSPEC (J. Wang et al. 2016, these proceedings).

4. Phase IV: New high-density DM (HODM), and coronagraphs optimized for segmented telescopes and single-object spectroscopy (G. Ruane et al. 2016, these proceedings).

\section{PERFORMANCE AND YIELD ESTIMATES}

To demonstrate the superiority of KPIC over current high contrast imaging capabilities in the regime of faint NGS in the visible, we ran extensive numerical simulations to estimate the yield of a notional large-scale 500-star survey (see Fig. 1 for more details about the sample).

Sensing in the near-IR versus visible vastly expands the volume of targets that can be addressed with extreme AO coronagraphy. This is illustrated in Fig. 3 which shows the current and predicted L-band performance on the left and a completeness determination for our notional survey based on an end-to-end model that includes an AO simulator, and Fresnel propagation through a realistic prescription of the Keck telescope, AO system and NIRC2 camera. The final contrast results include speckle, photon, background, and detector noise sources. Each target in the sample is passed through the model, producing a contrast curve which is interpolated by the BT-SETTL evolutionary model, producing detection limits in Jupiter mass units. Monte Carlo simulations are run to produce detection probability maps which are averaged to yield the contour plot. For spectral type M0V stars, the distance limit increases from $50 \mathrm{pc}$ to $150 \mathrm{pc}$ (30 times the volume). For late M-type stars, such as $\mathrm{M} 6 \mathrm{~V}$, the distance goes from $4 \mathrm{pc}$ to $100 \mathrm{pc}, 15,000$ times the volume!

The recently installed L-band vortex coronagraph of NIRC2 (KPIC phase I) will be used to perform high contrast imaging at the diffraction limit of the $10 \mathrm{~m}$ Keck telescope (inner working angle $\simeq 80$ mas). The new 
NIRC2 vortex mask is a phase-based coronagraph made from a concentric subwavelength grating etched in a diamond substrate. ${ }^{14,15}$ It offers well-demonstrated high contrast performance at a very small IWA, ${ }^{16,17}$ see also B. Femenia et al. 2016, O. Absil et al. 2016, E. Huby et al. 2016, and M. Bottom et al. 2016 (these proceedings).

L-band coronagraphy is the perfect complement to near-IR wavefront sensing for cooler M-stars because their red colors make them particularly bright in the L-band with respect to the background so that a coronagraph can yield its best starlight suppression factor. The dynamic range enabled by the L-band coronagraph allows reaching the planetary regime much faster around M-stars than earlier types. All in all, near-IR wavefront sensing plus L-band small IWA coronagraphy of late-type star is the ideal instrumental setup for exoplanet imaging.

\section{HIGH-RESOLUTION HIGH-CONTRAST SPECTROSCOPY}

Coupling a high-resolution spectrograph with a high-contrast imaging instrument (Fig. 4) is the next big step in the direct characterization of exoplanet atmospheres. ${ }^{11}$ In this scheme, the high-contrast imaging system serves as a spatial filter to separate the light from the star and the planet, and the high-resolution spectrograph serves as spectral filter, which differentiates between features in the stellar and planetary spectra, e.g., between different absorption lines and radial velocities. High-resolution spectroscopy has three game-changing benefits:

1. Detailed species-by-species molecular characterization (spectral retrieval).

2. Doppler measurements (planet spin, orbital velocity, plus mapping of atmospheric and/or surface features).

3. Last but not least, improved detection capability by side-stepping speckle noise calibration issues affecting low-resolution spectroscopic data from current integral field spectrographs such as SPHERE and GPI (Fig. 5).

Most direct imaging results so far have indeed focused on photometry and very low-resolution spectroscopy, due to the lack of instruments designed to optimally merge high contrast imaging (wavefront control and coronagraphy) and high-resolution spectroscopy at small angles from the host star. KPIC, and its fiber injection unit (FIU) linking Keck AO to NIRSPEC (Fig. 4) would enable routine high-resolution characterization of directly imaged low-mass companions and exoplanets. Fig. 5 shows the cross-correlation peak signal-to-noise ratio as a function of raw starlight suppression for the prototypical HR8799e exoplanet case, demonstrating that KPIC and its FIU can perform fine molecular composition retrieval under realistic levels of starlight suppression. The KPIC FIU to NIRSPEC will also make Doppler imaging mainstream. ${ }^{9}$ See J. Wang et al. 2016 (these proceedings) for more details.

\section{CONCLUSIONS AND PERSPECTIVES}

KPIC, with its IWA of $\simeq 25$ mas at J-band and $\simeq 80$ mas at L-band, will complement JWST at very small angles (the IWA of NIRCAM and MIRI will be 5-10 times larger). KPIC's high resolution spectroscopic mode will demonstrate crucial capabilities that will be essential to follow-up discoveries from JWST, TESS and later on WFIRST-AFTA. KPIC will bridge the gap between the highly productive Keck AO system and a TMT planet imager. While theoretical designs exist for segmented telescopes, there is still much to learn about coronagraphy on segmented telescopes, which is critical to future segmented telescopes in space (LUVOIR and possibly HabEx, see Roberge et al. 2016, and Mennesson et al. 2016, these proceedings). Keck provides the most credible pathfinder for ExAO on the highly-segmented TMT. KPIC will allow demonstrating critical componentlevel and system-level aspects, gain operational experience on segmented telescopes, and enable unique science, vetting the most promising targets for future follow-ups.

\section{ACKNOWLEDGMENTS}

This work was partially carried out at the Jet Propulsion Laboratory, California Institute of Technology, under contract with the National Aeronautics and Space Administration. 


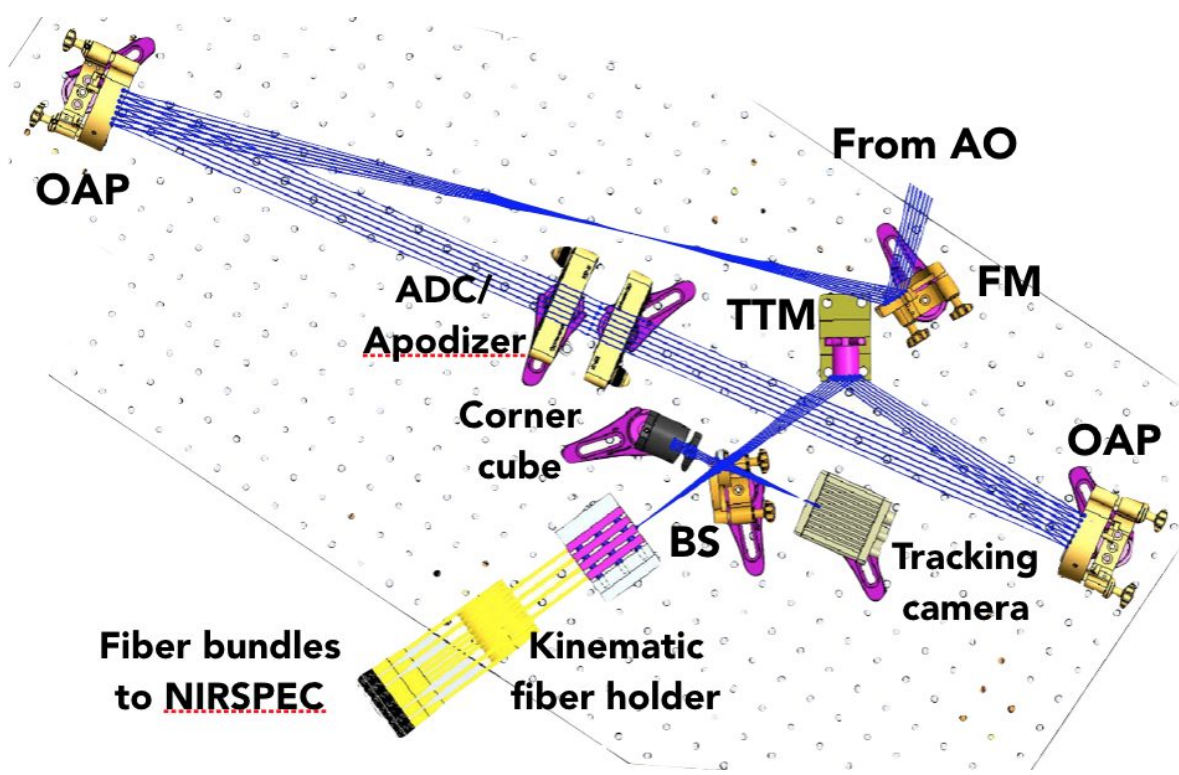

Figure 4. Notional layout for our Keck Fiber Injection Unit. The light from the AO system collimated by an off-axis parabola (OAP), directed to an Atmospheric Dispersion Compensator (ADC), apodizer coronagraphic combo (optimized to manage the starlight diffracted by the segmented Keck aperture), then refocussed by another OAP. The converging beam bounces off a tip-tilt mirror (TTM) then goes through a beam splitter and a lens to match the single-mode fiber NA. The light reflected off the beamsplitter (BS) is sent to a well-sampled, large field tracking camera. A reference fiber is retro-fed with a calibration lamp sending a beam backwards in the system, bouncing off the BS and collimated to the corner cube, then back on the tracking camera. The reference fiber is used to precisely register the position of the science fiber and beam, and track its position in real time.
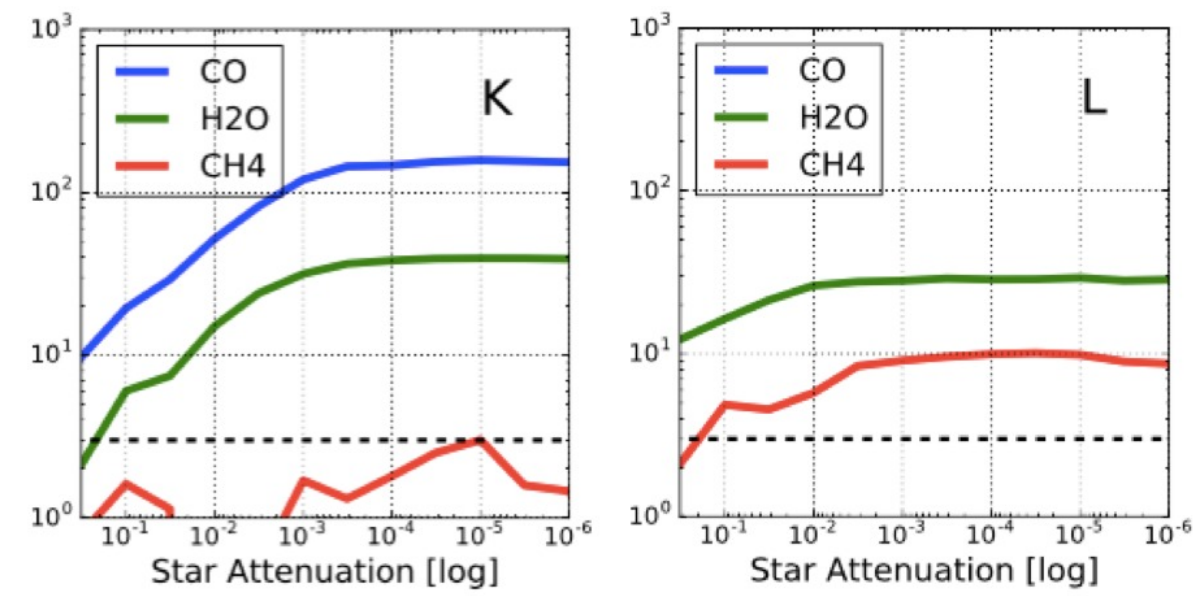

Figure 5. Signal to noise ratio of the cross correlation peak obtained by cross-correlating a high-resolution noiseless theoretical template of a given molecular species with the simulated $\mathrm{R}=37,500$ spectrum of HR8799e obtained with Keck/NIRSPEC, versus raw starlight attenuation. See J. Wang et al. 2016 (these proceedings) for additional details.

\section{REFERENCES}

[1] Kirkpatrick, J. D., Gelino, C. R., Cushing, M. C., Mace, G. N., Griffith, R. L., Skrutskie, M. F., Marsh, K. A., Wright, E. L., Eisenhardt, P. R., McLean, I. S., Mainzer, A. K., Burgasser, A. J., Tinney, C. G., Parker, S., and Salter, G., "Further Defining Spectral Type "Y" and Exploring the Low-mass End of the Field Brown Dwarf Mass Function," ApJ 753, 156 (July 2012).

[2] Johnson, J. A., Aller, K. M., Howard, A. W., and Crepp, J. R., "Giant Planet Occurrence in the Stellar Mass-Metallicity Plane," PASP 122, 905-915 (Aug. 2010). 
[3] Swift, J. J., Johnson, J. A., Morton, T. D., Crepp, J. R., Montet, B. T., Fabrycky, D. C., and Muirhead, P. S., "Characterizing the Cool KOIs. IV. Kepler-32 as a Prototype for the Formation of Compact Planetary Systems throughout the Galaxy," ApJ 764, 105 (Feb. 2013).

[4] Dressing, C. D. and Charbonneau, D., "The Occurrence Rate of Small Planets around Small Stars," ApJ 767, 95 (Apr. 2013).

[5] Petigura, E. A., Howard, A. W., and Marcy, G. W., "Prevalence of Earth-size planets orbiting Sun-like stars," Proceedings of the National Academy of Science 110, 19273-19278 (Nov. 2013).

[6] Bowler, B. P., Liu, M. C., Shkolnik, E. L., and Tamura, M., "Planets around Low-mass Stars (PALMS). IV. The Outer Architecture of M Dwarf Planetary Systems," ApJS 216, 7 (Jan. 2015).

[7] Cassan, A., Kubas, D., Beaulieu, J.-P., Dominik, M., Horne, K., Greenhill, J., Wambsganss, J., Menzies, J., Williams, A., Jørgensen, U. G., Udalski, A., Bennett, D. P., Albrow, M. D., Batista, V., Brillant, S., Caldwell, J. A. R., Cole, A., Coutures, C., Cook, K. H., Dieters, S., Prester, D. D., Donatowicz, J., Fouqué, P., Hill, K., Kains, N., Kane, S., Marquette, J.-B., Martin, R., Pollard, K. R., Sahu, K. C., Vinter, C., Warren, D., Watson, B., Zub, M., Sumi, T., Szymański, M. K., Kubiak, M., Poleski, R., Soszynski, I., Ulaczyk, K., Pietrzyński, G., and Wyrzykowski, Ł., "One or more bound planets per Milky Way star from microlensing observations," Nature 481, 167-169 (Jan. 2012).

[8] Benneke, B., "Strict Upper Limits on the Carbon-to-Oxygen Ratios of Eight Hot Jupiters from SelfConsistent Atmospheric Retrieval," arXiv.org, 7655 (Apr. 2015).

[9] Crossfield, I. J. M., Biller, B., Schlieder, J. E., Deacon, N. R., Bonnefoy, M., Homeier, D., Allard, F., Buenzli, E., Henning, T., Brandner, W., Goldman, B., and Kopytova, T., "A global cloud map of the nearest known brown dwarf," Nature 505, 654-656 (Jan. 2014).

[10] Snellen, I. A. G., Brandl, B. R., de Kok, R. J., Brogi, M., Birkby, J., and Schwarz, H., "Fast spin of the young extrasolar planet $\beta$ Pictoris b," Nature 509, 63-65 (May 2014).

[11] Snellen, I., de Kok, R., Birkby, J. L., Brandl, B., Brogi, M., Keller, C., Kenworthy, M., Schwarz, H., and Stuik, R., "Combining high-dispersion spectroscopy with high contrast imaging: Probing rocky planets around our nearest neighbors," A $\mathscr{E} A$ 576, A59 (Apr. 2015).

[12] Costa, J. B., Hippler, S., Feldt, M., Esposito, S., Ragazzoni, R., Bizenberger, P., Puga, E., and Henning, T. F. E., "PYRAMIR: a near-infrared pyramid wavefront sensor for the Calar Alto adaptive optics system," in [Adaptive Optical System Technologies II], Wizinowich, P. L. and Bonaccini, D., eds., Proc. SPIE 4839, 280-287 (Feb. 2003).

[13] Furlan, E., Luhman, K. L., Espaillat, C., D’Alessio, P., Adame, L., Manoj, P., Kim, K. H., Watson, D. M., Forrest, W. J., McClure, M. K., Calvet, N., Sargent, B. A., Green, J. D., and Fischer, W. J., "The Spitzer Infrared Spectrograph Survey of T Tauri Stars in Taurus," ApJS 195, 3 (July 2011).

[14] Mawet, D., Riaud, P., Absil, O., and Surdej, J., "Annular Groove Phase Mask Coronagraph," ApJ 633, 1191-1200 (Nov. 2005).

[15] Delacroix, C., Absil, O., Forsberg, P., Mawet, D., Christiaens, V., Karlsson, M., Boccaletti, A., Baudoz, P., Kuittinen, M., Vartiainen, I., Surdej, J., and Habraken, S., "Laboratory demonstration of a mid-infrared AGPM vector vortex coronagraph," A\& $A$ 553, A98 (May 2013).

[16] Mawet, D., Absil, O., Delacroix, C., Girard, J. H., Milli, J., O’Neal, J., Baudoz, P., Boccaletti, A., Bourget, P., Christiaens, V., Forsberg, P., Gonte, F., Habraken, S., Hanot, C., Karlsson, M., Kasper, M., Lizon, J.-L., Muzic, K., Olivier, R., Peña, E., Slusarenko, N., Tacconi-Garman, L. E., and Surdej, J., "L'-band AGPM vector vortex coronagraph's first light on VLT/NACO. Discovery of a late-type companion at two beamwidths from an F0V star," A $\mathscr{G} A$ 552, L13 (Apr. 2013).

[17] Absil, O., Milli, J., Mawet, D., Lagrange, A.-M., Girard, J., Chauvin, G., Boccaletti, A., Delacroix, C., and Surdej, J., "Searching for companions down to 2 AU from $\beta$ Pictoris using the L'-band AGPM coronagraph on VLT/NACO," A\&A 559, L12 (Nov. 2013). 